\title{
Threshold defect production in silicon determined by density functional theory molecular dynamics simulations
}

\author{
E. Holmström, A. Kuronen, and K. Nordlund \\ Department of Physics and Helsinki Institute of Physics, University of Helsinki, P.O. Box 64, Helsinki FIN 00014, Finland
}

(Received 30 April 2008; published 9 July 2008)

\begin{abstract}
We studied threshold displacement energies for creating stable Frenkel pairs in silicon using density functional theory molecular dynamics simulations. The average threshold energy over all lattice directions was found to be $36 \pm 2_{\mathrm{ST}} \mathrm{AT} \pm 2_{\mathrm{SY}} \mathrm{ST} \mathrm{eV}$, and thresholds in the directions $\langle 100\rangle$ and $\langle 111\rangle$ were found to be $20 \pm 2_{\text {SY ST }} \mathrm{eV}$ and $12.5 \pm 1.5_{\text {SY ST }} \mathrm{eV}$, respectively. Moreover, we found that in most studied lattice directions, a bond defect complex is formed with a lower threshold than a Frenkel pair. The average threshold energy for producing either a bond defect or a Frenkel pair was found to be $24 \pm 1_{\mathrm{ST}} \mathrm{AT} \pm 2_{\mathrm{SY} \text { ST }} \mathrm{eV}$.
\end{abstract}

DOI: 10.1103/PhysRevB.78.045202

PACS number(s): 61.72.Cc, 02.70.Ns, 61.80.Az

\section{INTRODUCTION}

The threshold displacement energy of a material $E_{d}$ is the minimum recoil energy that a lattice atom needs to become permanently displaced to an interstitial position, thus forming a stable Frenkel pair. ${ }^{1-3} E_{d}$ is the single most fundamental quantity in determining the primary state of radiation damage in any material. It can be used to determine directly the number of defects created by electron irradiation, and in many models it is used to estimate the number of defects created by high-energy ion or neutron irradiation. ${ }^{4,5}$

Knowing $E_{d}$ in silicon is essential not only for the obvious applications of the material in the manufacturing of semiconductor devices, ${ }^{6,7}$ but also because of contexts such as particle accelerators, where silicon elements in detectors are exposed to extensive hadron damage. ${ }^{8}$ In spite of this vast technological interest in the quantity and its extensive study during the last few decades, ${ }^{9-17} E_{d}$ is poorly known in the material. Experimental methods show a widely varying scale of results for $E_{d}$ in the range of $10-30 \mathrm{eV},{ }^{9-11,17}$ and simulations carried out previously using classical potentials show a similarly wide range of results. ${ }^{12-16}$ In particular, there is no established value for $E_{d \text {,ave }}^{\text {av }}$, the average threshold displacement energy over all lattice directions, ${ }^{3}$ which is used, e.g., to estimate the total amount of damage produced during ion implantation or hadron bombardment.

In the current work, we employ density functional theory ${ }^{18}$ (DFT) molecular dynamics (MD) simulations to study $E_{d}$. DFT methods are known to generally predict irradiation-related quantities in good agreement with experiment. ${ }^{19,20}$ Previous quantum-mechanical calculations of $E_{d}$ in silicon have focused on a few of the main lattice directions, ${ }^{21-23}$ even though it is well known that the average threshold is often much larger than the values in the principal directions. $^{2,3}$

Here, we calculate $E_{d}$ with SIESTA (Ref. 24) over all lattice directions. We find a global minimum of $12.5 \pm 1.5_{\mathrm{SY} \text { ST }} \mathrm{eV}$ for $E_{d}$, in excellent agreement with experiment. For the average threshold energy $E_{d \text {,ave }}^{\mathrm{av}, \mathrm{FP}}$ (for exact definition see Ref. 3) we find the value $36 \pm 2_{\mathrm{ST} \mathrm{AT}} \pm 2_{\mathrm{SY} \text { ST }} \mathrm{eV}$, which is clearly higher than even the highest commonly used values for the quantity. For most of the studied directions, the bond defect complex ${ }^{25-27}$ is created with a lower threshold energy than a Frenkel pair. We thus additionally report an average threshold energy $E_{d \text {,ave }}^{\mathrm{av}, \mathrm{BD} / \mathrm{FP}}$ of $24 \pm 1_{\mathrm{ST} \text { AT }} \pm 2_{\mathrm{SY} \text { ST }} \mathrm{eV}$ for creating either a bond defect or a Frenkel pair.

\section{METHOD}

Determining $E_{d}$ in a specific direction consists of simulating recoils in that direction with increasing energy until a permanent defect is obtained. ${ }^{3}$ Obtaining a reliable value for $E_{d \text {,ave }}^{\text {av }}$ demands the determination of $E_{d}$ in a number of randomly selected lattice directions, and therefore involves simulating a large number of individual recoil events in the lattice. Since DFT MD calculations are extremely heavy computationally, it was necessary to perform optimization of the simulation parameters in preparation for the task of determining $E_{d \text {,ave }}^{\text {av }}$ Fast classical interatomic potentials were first used to optimize the general simulation conditions, and then defect formation energies for the various types of basic point defects were calculated for optimizing the DFT parameters. The classical simulations were performed using the PARCAS (Ref. 28) simulation code with the Tersoff (Ref. 29) and Stillinger-Weber (Ref. 30) potentials, and the DFT MD was done using SIESTA as a force module to PARCAS.

The classical simulations were aimed at finding the minimum necessary size of the system and the minimum time that the simulation of one recoil event must encompass for producing reliable results. To optimize the system size, $E_{d \text {,ave }}^{\mathrm{av}}$ was calculated with a decreasing number of atoms in a cubical simulation cell, starting with 4096 atoms and ending at 64 atoms. Periodic boundary conditions and an initial temperature of $0 \mathrm{~K}$ were employed with all simulations throughout the work. $E_{d \text {,ave }}^{\text {av }}$ was found to diverge with both potentials at a cell size of about 100 atoms, and so the iteration was continued by considering noncubical simulation cells somewhat larger than this limit. The Stillinger-Weber potential gave a higher value for $E_{d \text {,ave }}^{\mathrm{av}}$ than Tersoff, and thus demanded a larger cell for reliable calculations than the latter potential. Hence, the finer iteration was completed using the Stillinger-Weber potential, and it was deduced that a simulation cell of 144 atoms with the $z$ axis of the unit cell oriented in the $\langle 111\rangle$ direction was the smallest suitable system for the 


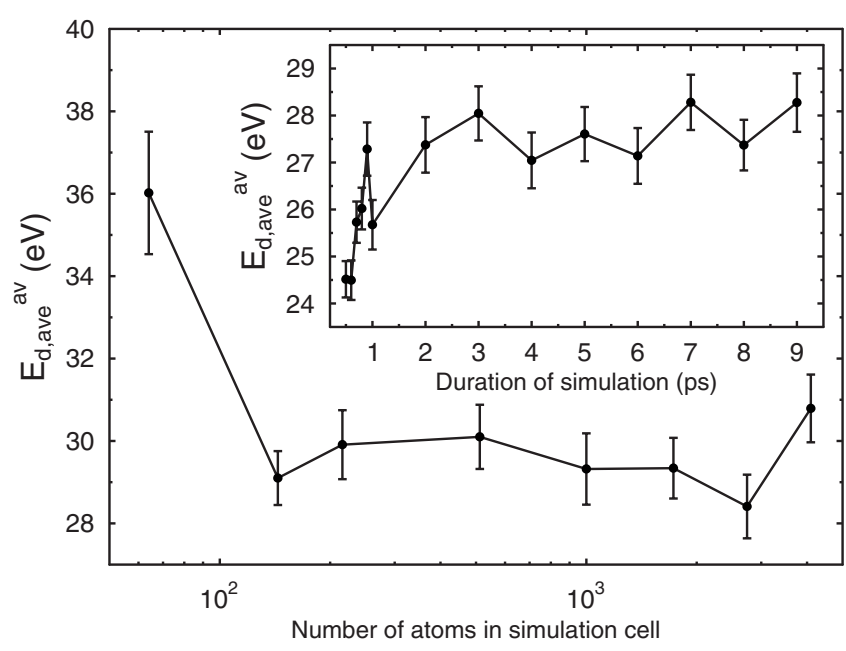

FIG. 1. Results for the scaling of the simulation cell size by calculating $E_{d \text {,ave }}^{\text {av }}$ with a simulation cell of a decreasing number of atoms using the Stillinger-Weber potential. $E_{d \text {,ave }}^{\text {av }}$ was found to diverge at a cell size below 100 atoms, and so the iteration was further continued with noncubical cells somewhat larger than this limit. Scaling of the simulation time is presented in the inset figure. It was found that $3000 \mathrm{fs}$ is a comfortably long simulation time for labeling the defects stable.

task of determining $E_{d \text {,ave }}^{\text {av }}$. Additionally, the symmetry of the diamond lattice was exploited by choosing recoil directions only in the positive octant of the standard unit cell for the atoms of both sublattices. Using the Stillinger-Weber potential, the largest cell size of 4096 yielded a value of $30.8 \pm 0.8_{\mathrm{ST} \text { AT }} \mathrm{eV}$ for $E_{d, \text { ave }}^{\mathrm{av}}$, and thus the value of $29.1 \pm 0.7_{\text {ST АT }} \mathrm{eV}$ given by the cell of 144 atoms implied a systematic error of $\pm 1.7 \mathrm{eV}$ in any determination of $E_{d, \text { ave }}^{\mathrm{av}}$ by DFT using the latter cell.

Different cooling methods for the periodic cell were also considered during the course of these initial simulations. Cooling the cell uniformly by Berendsen temperature scaling $^{31}$ with a time constant of $300 \mathrm{fs}$ after $200 \mathrm{fs}$ of the simulation had elapsed was found to be a method which did not significantly affect the result for $E_{d, \text { ave }}^{\text {av }}$ when compared to systems of larger sizes with border cooling only. The minimum necessary simulation time was then determined by calculating $E_{d \text {,ave }}^{\text {av }}$ with the 144 atom cell with a decreasing simulation time starting from the 10000 fs used thus far and ending at 500 fs. It was found that a simulation time of 3000 fs is comfortably long for labeling the defects stable. The results for the scaling of the cell size and the scaling of the simulation time are presented in Fig. 1.

The scaling of the DFT parameters was aimed at finding the computationally lightest possible parameter sets that would still provide good formation energies of the basic types of point defects in the lattice, in both the local-density approximation (LDA) and the generalized gradient approximation (GGA) for the exchange and correlation energies. It was hoped that calculations of $E_{d}$ within the GGA scheme would provide confirmation for calculations within the LDA scheme, which would be used for the heavy task of determining $E_{d \text {,ave }}^{\text {av }}$. Defect formation energies were considered an obvious test for the system, as the formation energy is a
TABLE I. Formation energies of the basic point defects in $\mathrm{eV}$ calculated within the chosen LDA scheme (see text) as a function of the number of $k$ points used in sampling the BZ. The values are missing for those defects that were not stable with respect to the conjugate gradient relaxation.

\begin{tabular}{ccccc}
\hline \hline $\begin{array}{c}\text { No. of } \\
k \text { points }\end{array}$ & Split $\langle 110\rangle$ & Hexagonal & Tetrahedral & Vacancy \\
\hline$\Gamma$ & 4.0 & - & - & 2.8 \\
4 & 4.7 & - & 5.7 & 3.4 \\
18 & 4.7 & 5.4 & 5.6 & 3.2 \\
\hline \hline
\end{tabular}

fundamental and much studied quantity in describing the entities which are the very object of the work. The varied parameters for the defect calculations were the LCAO basis set, the $k$-point sampling, and the equivalent plane-wave cutoff energy. The basis was varied between the single-zeta and double-zeta sets and each with polarization orbitals included, respectively. The number of $k$ points was scanned between a number of 18 and 4 points, and the single $\Gamma$ point. The equivalent plane-wave cutoff energy was varied between 50.0 to $300.0 \mathrm{Ry}$.

The defect formation energy is defined as

$$
E^{f}=\left(\frac{E_{d}}{N_{d}}-\frac{E_{u}}{N_{u}}\right) N_{d},
$$

where $E_{d}$ and $N_{d}$ are the potential energy and number of atoms in the defect cell, and $E_{u}$ and $N_{u}$ are the same in a defect-free cell. ${ }^{32}$ For each tested set of parameters, the lattice constant of the cell was first relaxed. This was then followed by the relaxation of the structures of the dumbbell or split- $\langle 110\rangle$ interstitial, the hexagonal interstitial, the tetrahedral interstitial, and the vacancy within a cell of the equilibrium lattice constant, using the conjugate gradient method. From these static calculations, it was found that only parameter sets utilizing the single-zeta basis could be considered in terms of computation times. Two such single-zeta sets, one within the LDA and one within the GGA approximation, were chosen for the molecular dynamics runs to come. Four $k$ points, a cutoff energy of $100.0 \mathrm{Ry}$, and the Ceperley-Alder exchange-correlation functional were used for the LDA set, and four $k$ points, a cutoff energy of $250.0 \mathrm{Ry}$, and the Perdew-Burke-Ernzerhof exchange-correlation functional were used for the GGA set. Tables I and II present the formation energies of the basic point defects for the LDA and the GGA set, respectively, as a function of the number of $k$ points that were used in sampling the Brillouin zone (BZ). It can be seen that the interstitial formation energies converge well, but there is a slight discrepancy in the vacancy formation energy for both sets. The formation energies of the aforementioned defects as well as results from other DFT studies are presented in Table III. Additionally, the groundstate Frenkel pair, which was found to consist of a tetrahedral interstitial and a close vacancy, and the bond defect, are included. In the LDA set, the hexagonal interstitial was not stable with respect to the relaxation, and instead relaxed toward a split configuration. There is considerable uncertainty 
TABLE II. Formation energies of the basic point defects in $\mathrm{eV}$ calculated within the chosen GGA scheme (see text) as a function of the number of $k$ points used in sampling the BZ. The calculation of the formation energy of the vacancy using only the $\Gamma$ point failed to converge.

\begin{tabular}{ccccc}
\hline \hline $\begin{array}{c}\text { No. of } \\
k \text { points }\end{array}$ & Split $\langle 110\rangle$ & Hexagonal & Tetrahedral & Vacancy \\
\hline$\Gamma$ & 4.3 & 4.3 & 4.8 & - \\
4 & 4.9 & 5.6 & 5.8 & 3.9 \\
18 & 4.9 & 5.6 & 5.8 & 3.6 \\
\hline \hline
\end{tabular}

about the interstitial formation energies. Our values are somewhat larger than those obtained in other recent studies, but the order in energy is similar. ${ }^{25,33,35}$ In particular, most studies indicate that the dumbbell is the ground state, in agreement with our result. ${ }^{27,34}$ The present results for interstitial formation energies conform to some of the earliest DFT calculations of point defects in silicon, where the tetrahedral and hexagonal interstitials were found to have formation energies of 5 to $6 \mathrm{eV}$ and 4 to $5 \mathrm{eV}$, respectively. ${ }^{35-38}$ The formation enthalpy of a Frenkel pair consisting of a tetrahedral interstitial and a vacancy was recently found to be lower than that of the corresponding pair with a dumbbell interstitial, the dumbbell however being the isolated groundstate interstitial, as is the case here. ${ }^{39}$

Finally, as a separate test for the used Troullier-Martins pseudopotential, the potential energy of the silicon dimer was computed for distances of $r=0.8$ to $10 \AA$ within the LDA and GGA schemes and compared to all-electron calculations. The result was that reliable calculations could be performed up to energies of at least $100 \mathrm{eV},{ }^{40}$ which should easily suffice for calculations of $E_{d}$ in any lattice direction.

After the tests were finalized, $E_{d}$ was calculated for a total of 80 random directions for atoms of both sublattices within the LDA scheme, and for 20 random directions within the GGA scheme. Additionally, $E_{d}$ was calculated for the lattice directions $\langle 111\rangle$ and $\langle 100\rangle$.

TABLE III. Formation energies of the basic point defects in $\mathrm{eV}$ calculated within the chosen LDA and GGA schemes, and results from other DFT studies. The ground-state Frenkel pair for our calculations consists of a tetrahedral interstitial and a vacancy with a distance of $4.7 \AA$ between them. No formation energy for this defect was found in the literature.

\begin{tabular}{cccc}
\hline \hline Defect & LDA & GGA & Other studies \\
\hline Split $\langle 110\rangle$ & 4.7 & 4.9 & $2.88-3.84^{25,27,33,34}$ \\
Hexagonal & - & 5.6 & $2.87-3.80^{25,27,33,34}$ \\
Tetrahedral & 5.7 & 5.8 & $3.43-5.1^{33,25}$ \\
Vacancy & 3.4 & 3.9 & $3.17-3.65^{25,27,34,41}$ \\
Frenkel pair & 6.8 & 7.5 & - \\
Bond defect & 2.6 & 3.0 & $2.34-2.80^{27,34}$ \\
\hline \hline
\end{tabular}

TABLE IV. Threshold displacement energies in eV calculated within the LDA and GGA schemes. The energy step size was $1 \mathrm{eV}$ for the direction specific thresholds, implying an error of $\pm 0.5 \mathrm{eV}$ in addition to the systematic error of $\pm 1.0 \mathrm{eV}$ resulting from the scaling of the cell size. For the averages, the error consists of the statistical standard error and the systematic error of $\pm 2 \mathrm{eV}$ resulting from the scaling of the cell size. $A$ and $B$ denote the closed and open $\langle 111\rangle$ directions, respectively.

\begin{tabular}{ccc}
\hline \hline & LDA & GGA \\
\hline$\langle 111\rangle(A)$ & $14.5 \pm 1.5_{\mathrm{SY} \mathrm{ST}}$ & $14.5 \pm 1.5_{\mathrm{SY} \mathrm{ST}}$ \\
$\langle 111\rangle(B)$ & $12.5 \pm 1.5_{\mathrm{SY} \mathrm{ST}}$ & $12.5 \pm 1.5_{\mathrm{SY} \mathrm{ST}}$ \\
$\langle 100\rangle$ & $20.5 \pm 1.5_{\mathrm{SY} \mathrm{ST}}$ & $19.5 \pm 1.5_{\mathrm{SY} \mathrm{ST}}$ \\
$E_{d, \mathrm{ave}}^{\mathrm{av}, \mathrm{FP}}$ & $36 \pm 2_{\mathrm{ST} \mathrm{AT}} \pm 2_{\mathrm{SY} \mathrm{ST}}$ & $35 \pm 4_{\mathrm{ST} \mathrm{AT}} \pm 2_{\mathrm{SY} \mathrm{ST}}$ \\
$E_{d, \text { ave }}^{\text {av, }}$ & $24 \pm 1_{\mathrm{ST} \mathrm{AT}} \pm 2_{\mathrm{SY} \mathrm{ST}}$ & $23 \pm 2_{\mathrm{ST} \mathrm{AT}} \pm 2_{\mathrm{SY} \mathrm{ST}}$ \\
\hline \hline
\end{tabular}

\section{RESULTS}

The results for the threshold displacement energies calculated within the LDA and GGA schemes are presented in Table IV. A large number of bond defects was observed throughout the simulations, which somewhat complicated the determination of the global minimum as well as the average threshold energy. The results for the directions $\langle 111\rangle$ and $\langle 100\rangle$ given above are the threshold energies required for producing a defect of any type in those directions, either a Frenkel pair or a bond defect, and in these directions the lower threshold energy was for the Frenkel pair. However, bond defects were observed for a recoil energy as low as 12 $\mathrm{eV}$ in some random directions, and hence we determined an average threshold energy $E_{d \text {,ave }}^{\mathrm{av}, \mathrm{BD} / \mathrm{FP}}$ for producing either a bond defect or a Frenkel pair in addition to the average threshold energy for producing a Frenkel pair, $E_{d, \text { ave }}^{\mathrm{av}, \mathrm{FP}}$.

We obtained values of $36 \pm 2_{\mathrm{ST} \mathrm{AT}} \pm 2_{\mathrm{SY}} \mathrm{ST}$ and $35 \pm 4_{\mathrm{ST} \mathrm{AT}} \pm 2_{\mathrm{SY} \mathrm{ST}} \mathrm{eV}$ for $E_{d \text {,ave }}^{\mathrm{av}, \mathrm{FP}}$ within the LDA and GGA schemes, respectively, and $24 \pm 1_{\mathrm{ST} \mathrm{AT}} \pm 2_{\mathrm{SY} \text { ST }}$ and $23 \pm 2_{\mathrm{ST} \mathrm{AT}} \pm 2_{\mathrm{SY} \text { ST }} \mathrm{eV}$ for $E_{d \text {,ave }}^{\mathrm{av}, \mathrm{BD} / \mathrm{FP}}$. The LDA and GGA values are sufficiently close for the LDA values, computed from a greater number of directions, to be considered statistically reliable. In the $\langle 111\rangle$ direction, we found a value of $12.5 \mathrm{eV}$ for $E_{d}^{\mathrm{FP}}$, the threshold energy for producing Frenkel pairs, and since no value lower than $12.5 \mathrm{eV}$ for $E_{d}^{\mathrm{FP}}$ was obtained in any of the directions studied throughout the simulations, we take $12.5 \mathrm{eV}$ to be the global minimum of $E_{d}^{\mathrm{FP}}$. This is in excellent agreement with the experimental findings that Frenkel pair production in the $\langle 111\rangle$ direction of the silicon lattice is greater than in the other two main lattice directions, $\langle 100\rangle$ and $\langle 110\rangle$. $^{11,42}$

For both the GGA and LDA runs, the tetrahedral interstitial type accounts for about $75 \%$ of all the Frenkel pair end state configurations, which is consistent with the finding that the ground-state Frenkel pair includes a tetrahedral interstitial. The typical formation kinetics of this type of Frenkel pair invoke a replacement collision chain of the atoms in the $\langle 111\rangle$ direction, as illustrated in Fig. 2. Here, atom 1 is given an initial recoil of $15 \mathrm{eV}$ in the $\langle 111\rangle$ direction directly toward atom 2, which results in atom 2 being pushed forward from its site and atom 1 rebounding backward. Atom 2 con- 

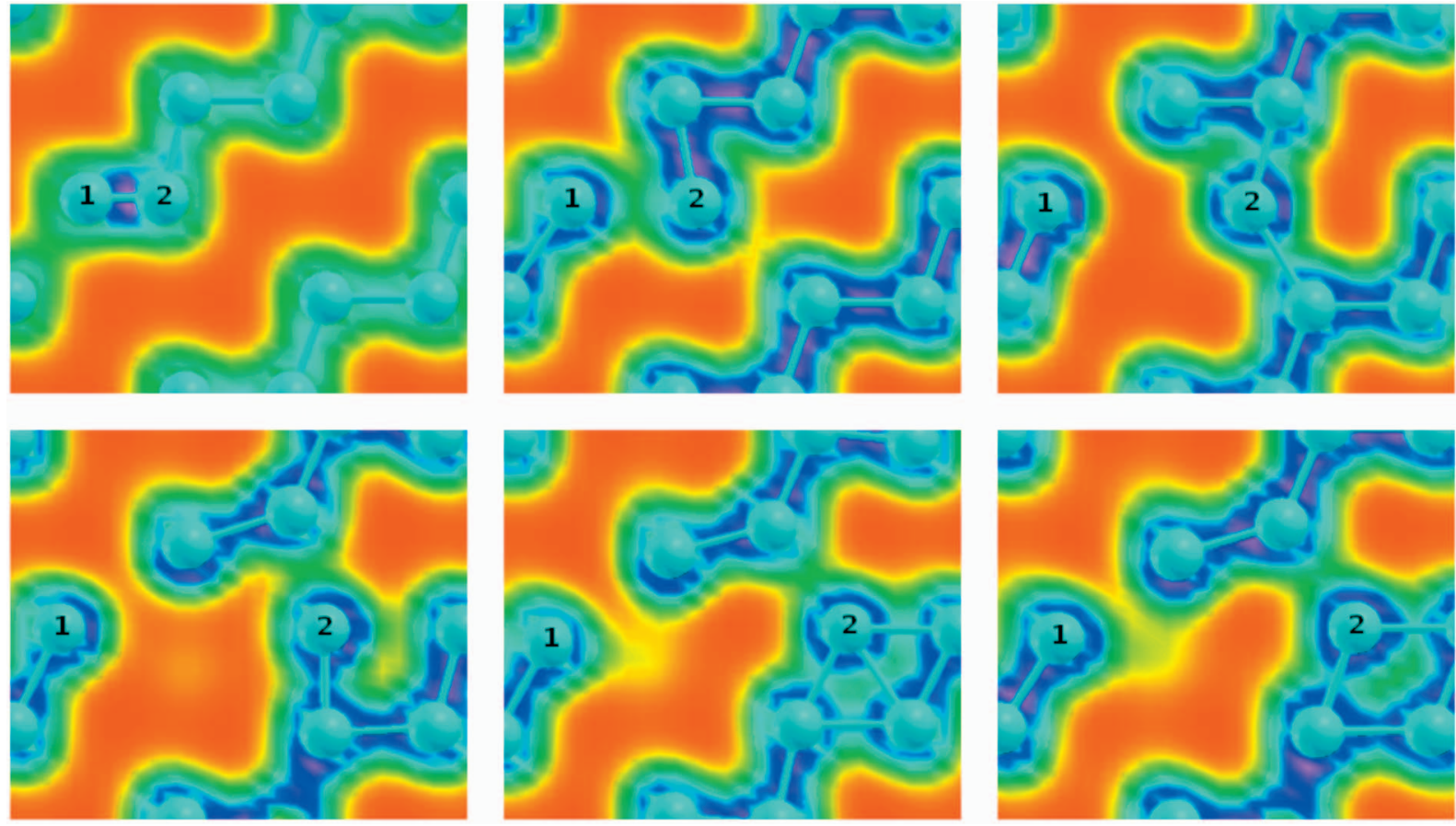

FIG. 2. (Color) Illustration (Ref. 43) of the electron density in a [1 $\overline{1} 0]$ plane around a replacement collision sequence that is typically observed for recoils in the $\langle 111\rangle$ direction resulting in a close Frenkel pair. Atom 1 is initially given a recoil in the $\langle 111\rangle$ direction, which results in atom 2 falling into a tetrahedral interstitial position, leaving a vacancy behind at its original lattice site. Red indicates low electron density.

tinues in the $\langle 111\rangle$ direction until it settles into a tetrahedral interstitial position, and a vacancy is left behind at the original site of the atom. The second type of observed end state interstitial is the dumbbell state, which was observed for $15 \%$ of all Frenkel pair end states. The remaining $10 \%$ of Frenkel pair end states are characterized by a small cluster of vacancies and interstitials, instead of clearly separable point defects. In some $60 \%$ of all the directions studied, a bond defect complex was created with a lower threshold energy than a Frenkel pair. This is to be expected, as the formation energy of this defect was found to be considerably lower than that of the Frenkel pair.

\section{DISCUSSION}

The result of $12.5 \pm 1.5_{\mathrm{SY}} \mathrm{ST} \mathrm{eV}$ for the global minimum of $E_{d}^{\mathrm{FP}}$ is in excellent agreement with the experimental value of $12.9 \pm 0.6 \mathrm{eV}$ obtained by Loferski and Rappaport ${ }^{9}$ for the onset of damage in silicon under electron irradiation. Additionally, our result of $20 \pm 2_{\mathrm{SY} \mathrm{ST}} \mathrm{eV}$ for $E_{d}^{\mathrm{FP}}$ in the $\langle 100\rangle$ direction is in excellent agreement with the frequently quoted value of $21 \mathrm{eV}$ from a study by Corbett and Watkins ${ }^{10}$ where the orientation of the electron beam was along a $\langle 100\rangle$ axis. The present results are systematically somewhat higher but in fair agreement with previous quantum-mechanical calculations by DFT and tight-binding molecular dynamics. ${ }^{22,23}$ The obtained value of $36 \pm 2_{\mathrm{ST} \mathrm{AT}} \pm 2_{\mathrm{SY} \text { ST }} \mathrm{eV}$ for $E_{d \text {, ave }}^{\mathrm{av}, \mathrm{FP}}$ is closer to the Stillinger-Weber average of $30.8 \pm 0.8_{\text {ST }}$ AT $\mathrm{eV}$ than the Tersoff average of $18.9 \pm 0.6_{\mathrm{ST}}$ AT $\mathrm{eV}$ obtained with the 4096 atom simulation cells. However, the direc- tional thresholds of $20.5 \pm 0.5_{\mathrm{SY} \text { ST }} \mathrm{eV}$ for $\langle 111\rangle(A)$, $16.5 \pm 0.5_{\mathrm{SY}} \mathrm{ST} \mathrm{eV}$ for $\langle 111\rangle(B)$, and $23.5 \pm 0.5_{\mathrm{SY}} \mathrm{ST} \mathrm{eV}$ for $\langle 100\rangle$ given by the Stillinger-Weber potential greatly overshoot the present DFT results with the only fair agreement being in the $\langle 100\rangle$ direction.

It should be noted, that the bond defect is invisible to standard experimental techniques, ${ }^{27}$ and therefore a comparison to experiment of our results concerning bond defects cannot be made at the present. However, our observation of large numbers of bond defects is in excellent agreement with the very recent experimental deduction that the bond defect plays a significant role in the amorphization of silicon. ${ }^{44}$

Values used for $E_{d \text {,ave }}^{\mathrm{av}, \mathrm{in}}$ in the radiation effects and material damage community appear in the range of 13 to $25 \mathrm{eV}^{8,45-47}$ The common usage of 13 and $15 \mathrm{eV}$ for the value of the parameter is highly inappropriate, as from our simulations it is clear that the actual value is over a factor of two higher. For example, the usage of $36 \mathrm{eV}$ in place of these two lower values in the widely used Kinchin-Pease model ${ }^{4}$ results in the direct scaling of the number of defects created by an energetic ion by more than a factor of two. As another example, the SRIM2003 code ${ }^{46}$ based on the BCA approximation gives the number of vacancies created by a $10 \mathrm{keV} \mathrm{Si}$ implantation of $\mathrm{Si}$ as 273, 238, and 101 for the values of 13, 15 , and $36 \mathrm{eV}$ for $E_{d \text {,ave }}^{\mathrm{av}, \mathrm{FP}}$, respectively. Even the highest community value of $25 \mathrm{eV}$ appears too low an average threshold displacement energy as input for a radiation damage model. Finally, we note that the current data spanning all lattice directions could also be used in crystal BCA codes such as MARLOWE (Ref. 48) as input for direction specific threshold displacement energies. 


\section{CONCLUSIONS}

In summary, using DFT molecular dynamics simulations, we have determined the average threshold displacement energy for creating Frenkel pairs in silicon to be $36 \pm 2_{\text {ST AT }} \pm 2_{\text {SY ST }} \mathrm{eV}$. The global minimum of the threshold displacement energy was found to be 12.5 $\pm 1.5_{\mathrm{SY}} \mathrm{sT} \mathrm{eV}$, in the $\langle 111\rangle$ direction, which is in excellent agreement with experiment. Our results show that $E_{d \text {,ave }}^{\mathrm{av}, \mathrm{FP}}$ is clearly higher than the values used commonly in ion irradia- tion damage models. We find additionally, that a bond defect complex is formed in most lattice directions with a lower threshold energy than a Frenkel pair.

\section{ACKNOWLEDGMENTS}

The research was supported by the Academy of Finland Center of Excellence in Computational Molecular Science. Grants of computer time from the Center for Scientific Computing in Espoo, Finland are gratefully acknowledged.
${ }^{1} \mathrm{P}$. Lucasson, in Fundamental Aspects of Radiation Damage in Metals, edited by M. T. Robinson and F. N. Young, Jr. (ORNL, Springfield, 1975), pp. 42-65.

${ }^{2}$ P. Jung, Production of Atomic Defects in Metals, LandoltBörnstein, New Series, Group III, Vol. 25 (Springer, Berlin, 1991), Chap. 1, pp. 1-86.

${ }^{3}$ K. Nordlund, J. Wallenius, and L. Malerba, Nucl. Instrum. Methods Phys. Res. B 246, 322 (2006).

${ }^{4}$ G. Kinchin and R. Pease, Rep. Prog. Phys. 18, 1 (1955).

${ }^{5}$ J. P. Biersack and L. G. Haggmark, Nucl. Instrum. Methods 174, 257 (1980).

${ }^{6}$ J. F. Gibbons, Proc. IEEE 56, 295 (1968).

${ }^{7}$ J. W. Mayer and S. S. Lau, Electronic Materials Science for Integrated Circuits in Si and GaAs (MacMillan, New York, 1990).

${ }^{8}$ M. Huhtinen, Nucl. Instrum. Methods Phys. Res. A 491, 194 (2002).

${ }^{9}$ J. Loferski and P. Rappaport, Phys. Rev. 111, 432 (1958).

${ }^{10}$ J. Corbett and G. D. Watkins, Phys. Rev. 138, A555 (1965).

${ }^{11}$ P. Hemment and P. Stevens, J. Appl. Phys. 40, 4893 (1969).

${ }^{12}$ L. A. Miller, D. K. Brice, A. K. Prinja, and S. T. Picraux, Phys. Rev. B 49, 16953 (1994).

${ }^{13}$ L. Miller, D. Brice, A. Prinja, and T. Pricraux, in Defects in Materials, MRS Symposia Proceedings No. 209, edited by P. D. Bristowe, I. E. Epperson, I. E. Griffith, and Z. Liliental-Weber (Materials Research Society, Pittsburgh, 1991), p. 171.

${ }^{14}$ L. Miller, D. Brice, A. Prinja, and T. Pricraux, Radiat. Eff. Defects Solids 129, 127 (1994).

${ }^{15}$ M.-J. Caturla, T. Diaz de la Rubia, and G. H. Gilmer, in Materials Synthesis and Processing Using Ion Beams, MRS Symposia Proceedings No. 316, edited by R. I. Culbertson, O. W. Holland, K. S. Jones, and K. Maex (Materials Research Society, Pittsburgh, 1994), p. 141.

${ }^{16}$ M. Sayed, J. H. Jefferson, A. B. Walker, and A. G. Cullis, Nucl. Instrum. Methods Phys. Res. B 102, 232 (1995).

${ }^{17}$ D. Marton, in Low Energy Ion-Surface Interactions, edited by J. W. Rabalais (Wiley, Chester, 1994), p. 526.

${ }^{18}$ For a review, see R. O. Jones and O. Gunnarsson, Rev. Mod. Phys. 61, 689 (1989).

${ }^{19}$ B. Sadigh, T. J. Lenosky, S. K. Theiss, M.-J. Caturla, T. Diaz de la Rubia, and M. A. Foad, Phys. Rev. Lett. 83, 4341 (1999).

${ }^{20}$ C.-C. Fu, J. D. Torre, F. Willaime, and J.-L. Bocquet, Nat. Mater. 4, 68 (2005).

${ }^{21}$ S. Uhlmann, T. Frauenheim, K. Boyd, D. Marton, and J. Rabalais, Radiat. Eff. Defects Solids 141, 185 (1997).
${ }^{22}$ W. Windl, T. J. Lenosky, J. D. Kress, and A. F. Voter, Nucl. Instrum. Methods Phys. Res. B 141, 61 (1998).

${ }^{23}$ M. Mazzarolo, L. Colombo, G. Lulli, and E. Albertazzi, Phys. Rev. B 63, 195207 (2001).

${ }^{24}$ J. M. Soler, E. Artacho, J. D. Gale, A. García, J. Junquera, P. Ordejón, and D. Sánchez-Portal, J. Phys.: Condens. Matter 14, 2745 (2002).

${ }^{25}$ M. Tang, L. Colombo, J. Zhu, and T. Diaz de la Rubia, Phys. Rev. B 55, 14279 (1997).

${ }^{26}$ F. Cargnoni, C. Gatti, and L. Colombo, Phys. Rev. B 57, 170 (1998).

${ }^{27}$ S. Goedecker, T. Deutsch, and L. Billard, Phys. Rev. Lett. 88, 235501 (2002).

${ }^{28} \mathrm{~K}$. Nordlund, PARCAS computer code, 2006. The main principles of the molecular dynamics algorithms are presented in Refs. 49 and 50. The adaptive time step and electronic stopping algorithms are the same as in Ref. 51.

${ }^{29}$ J. Tersoff, Phys. Rev. B 38, 9902 (1988).

${ }^{30}$ F. H. Stillinger and T. A. Weber, Phys. Rev. B 31, 5262 (1985).

${ }^{31}$ H. J. C. Berendsen, J. P. M. Postma, W. F. van Gunsteren, A. DiNola, and J. R. Haak, J. Chem. Phys. 81, 3684 (1984).

${ }^{32}$ G.-X. Qian, R. M. Martin, and D. J. Chadi, Phys. Rev. B 38, 7649 (1988).

${ }^{33}$ W.-K. Leung, R. J. Needs, G. Rajagopal, S. Itoh, and S. Ihara, Phys. Rev. Lett. 83, 2351 (1999).

${ }^{34}$ O. K. Al-Mushadani and R. J. Needs, Phys. Rev. B 68, 235205 (2003).

${ }^{35}$ Y. Bar-Yam and J. D. Joannopoulos, Phys. Rev. Lett. 52, 1129 (1984).

${ }^{36}$ R. Car, P. J. Kelly, A. Oshiyama, and S. T. Pantelides, Phys. Rev. Lett. 52, 1814 (1984).

${ }^{37}$ R. Car, P. J. Kelly, A. Oshiyama, and S. T. Pantelides, Phys. Rev. Lett. 54, 360 (1985).

${ }^{38}$ G. A. Baraff and M. Schluter, Phys. Rev. B 30, 3460 (1984).

${ }^{39}$ S. A. Centoni, B. Sadigh, G. H. Gilmer, T. J. Lenosky, T. Diaz de la Rubia, and C. B. Musgrave, Phys. Rev. B 72, 195206 (2005).

${ }^{40}$ DMOL is a trademark of AccelRys., Inc.

${ }^{41}$ M. J. Puska, S. Pöykkö, M. Pesola, and R. M. Nieminen, Phys. Rev. B 58, 1318 (1998).

${ }^{42}$ V. S. Vavilov, V. M. Patskevich, B. Y. Yurkov, and P. Y. Glazunov, Fiz. Tverd. Tela (Leningrad) 2, 1431 (1960).

${ }^{43}$ A. Kokalj, J. Mol. Graphics Modell. 17, 176 (1999).

${ }^{44}$ P. D. Edmondson, D. Riley, R. C. Birtcher, and S. E. Donnelly (unpublished).

${ }^{45}$ G. P. Summers, E. A. Burke, and R. J. Walters, IEEE Trans. 
Nucl. Sci. 40, 1372 (1993).

${ }^{46}$ J. F. Ziegler, SRIM-2003 software package, available online at http://www.srim.org

${ }^{47}$ R. E. MacFarlane, NJOY 99 Nuclear Data Processing System, code available at http://t2.lanl.gov/codes/njoy99/

${ }^{48}$ M. T. Robinson, Phys. Rev. B 40, 10717 (1989).
${ }^{49}$ K. Nordlund, M. Ghaly, R. S. Averback, M. Caturla, T. Diaz de la Rubia, and J. Tarus, Phys. Rev. B 57, 7556 (1998).

${ }^{50}$ M. Ghaly, K. Nordlund, and R. S. Averback, Philos. Mag. A 79, 795 (1999).

${ }^{51}$ K. Nordlund, Comput. Mater. Sci. 3, 448 (1995). 